\title{
Melt Conditioned Direct Chill Casting (MC-DC) Process for Production of High Quality Aluminium Alloy Billets
}

\author{
Jayesh B Patel ${ }^{1, a^{*}}$, Hu-Tian Li ${ }^{1}$, Xia Mingxu ${ }^{1}$, Simon Jones ${ }^{1}$, \\ Sundaram Kumar ${ }^{2}$, Keyna O'Reilly ${ }^{2}$ and Zhongyun Fan ${ }^{1}$ \\ ${ }^{1}$ The EPSRC Centre, LiME, BCAST, Brunel University, Uxbridge, UB8 3PH, UK \\ ${ }^{2}$ The EPSRC Centre, LiME, Department of Materials, University of Oxford, OX1 3PH, UK \\ ajayesh.patel@brunel.ac.uk
}

Keywords: Direct chill casting, Intensive melt shearing, Grain refinement, Solidification

\begin{abstract}
A novel direct chill (DC) casting process, melt conditioned direct chill (MC-DC) casting process, has been developed for production of high quality aluminium alloy billets. In the MC-DC casting process, a high shear device is submerged in the sump of the DC mould to provide intensive melt shearing, which in turn, disperses potential nucleating particles, creates a macroscopic melt flow to uniformly distribute the dispersed particles, and maintains a uniform temperature and chemical composition throughout the melt in the sump. Experimental results have demonstrated that, the MC-DC casting process can produce aluminium alloy billets with significantly refined microstructure and reduced cast defects. In this paper, we give an overview of the MC-DC casting process and report on results obtained from an industrial scale trial.
\end{abstract}

\section{Introduction}

Aluminium alloys are widely used in various industrial sectors such as automotive, construction and even electronic applications for the purpose of weight saving [1]. In order to produce high quality aluminum castings, it is important to be able to control the cast structure. It is well known that a fine and equiaxed grain structure facilitates the casting process for feedstock production, enhances the mechanical performance of shape casting, and improves extrudability, machinability and surface finish during downstream processing [2]. The production of wrought aluminium alloys starts with direct chill (DC) casting to produce billets or slabs of convenient shape and size for thermomechanical processing such as forging, extrusion or rolling in order to produce semi-finished products. Some of the problems encountered in conventional DC casting are formation of coarse and non-uniform microstructure, severe chemical segregation, porosity and hot tearing, which have a detrimental effect on rollability and extrudability and in turn result in poor mechanical properties of both semi-fabricated and finished products [2]. Achieving a fine and equiaxed grain structure, whilst preventing cracks and hot tearing, is typically achieved by chemical grain refinement [3]. Grain refinement by chemical inoculation is a process where potent nucleation sites are fed into a melt during casting, typically in the form of $\mathrm{Al}-\mathrm{Ti}-\mathrm{B}$ based master alloy rods for Al-alloys, and is the most widely used method of grain size control in industry. These heterogeneous nucleation sites effectively enhance heterogeneous nucleation of primary $\alpha$-Al grains and promote columnar to equiaxed transition during DC casting. This enables a fine and equiaxed microstructure and therefore a reduced tendency for macro-segregation and hot tearing. Grain refining of wrought aluminium alloys with $\mathrm{Al}-\mathrm{Ti}-\mathrm{B}$ master alloys is thought to be optimised now, however some disadvantages such as increased cost and potential for inclusions still persists [3]. Therefore, alternative technologies such as ultrasonic cavitation, electromagnetic stirring and intensive melt shearing $[4,5]$ are being investigated for improving cast quality. In this paper, we give an overview of the newly developed melt conditioned direct chill (MC-DC) casting process for the production of Al-alloy billets with improved quality, and report on results obtained from an industrial scale trial of the MC-DC casting process. The discussion will be focused on the solidification process under intensive melt shearing and outline the advantages of the MC-DC casting process. 


\section{The MC-DC process}

The melt conditioned DC casting (MC-DC) process is shown schematically in Fig.1. Intensive melt shearing for melt treatment can be achieved in a rotor-stator unit [6]. This unit comprises of a set of rotor and stator attached to an electrical motor with a speed control. During its operation, the motor passes the power to the rotor by the shaft and drives the rotor to rotate and shear the liquid metal in the gap between the rotor and the stator and also in the openings of the stator. The rotation speed can be in the order of 5000-15000rpm providing a shear rate of up to $10^{5} \mathrm{~s}^{-1}$. The new high shear device provides macro-flow in a volume of melt for distributive mixing and intensive shearing near the tip of the device for dispersive mixing. The main advantages of the high shear device include significantly enhanced kinetics for chemical reactions or phase transformations, uniform dispersion, distribution and size reduction of solid particles and gas bubbles, improved homogenisation of chemical composition and temperature fields and also forced wetting of usually difficult-to-wet solid particles in the liquid metal. In the MC-DC process, the high shear rotor-stator unit is submerged in the sump of a conventional DC mould to provide intensive melt shearing during casting. During operation, liquid metal is sucked into the high shear device and is subjected to intensive shearing between the rotor and stator and then forced through the holes on the stator as fine jet at very high velocity. The escaping liquid forms a unique macroscopic flow pattern in the sump, as shown in Fig. 1. The sucking action of the device induces a macroscopic flow in the lower part of the sump, which is much more intensive than that in the upper part of the sump. A major advantage of the high shear device is that it offers a useful combination of both dispersive and distributive mixing actions while keeping the melt surface relatively stable so as to avoid any possibility of entrainment of oxides and increased hydrogen intake from the atmosphere. Application of high shear during DC casting can have the following effects:

- The dispersive mixing action will disperse inclusions and oxide films in the melt into fine and individual particles (usually on nano scale), reducing/eliminating their usual harmful effects on the casting processes and quality of castings,

- The distributive mixing action will ensure the melt in the sump has a uniform temperature (close to the liquidus temperature of the alloy being cast), homogeneous chemical composition and well dispersed and uniformly distributed heterogeneous nucleation sites,

- The interaction between the macroscopic melt flow, i.e., the laminar flow paths as shown in Fig. 1 and the solidification front provides a flatter and shallower sump profile and the potential for increasing casting speed.

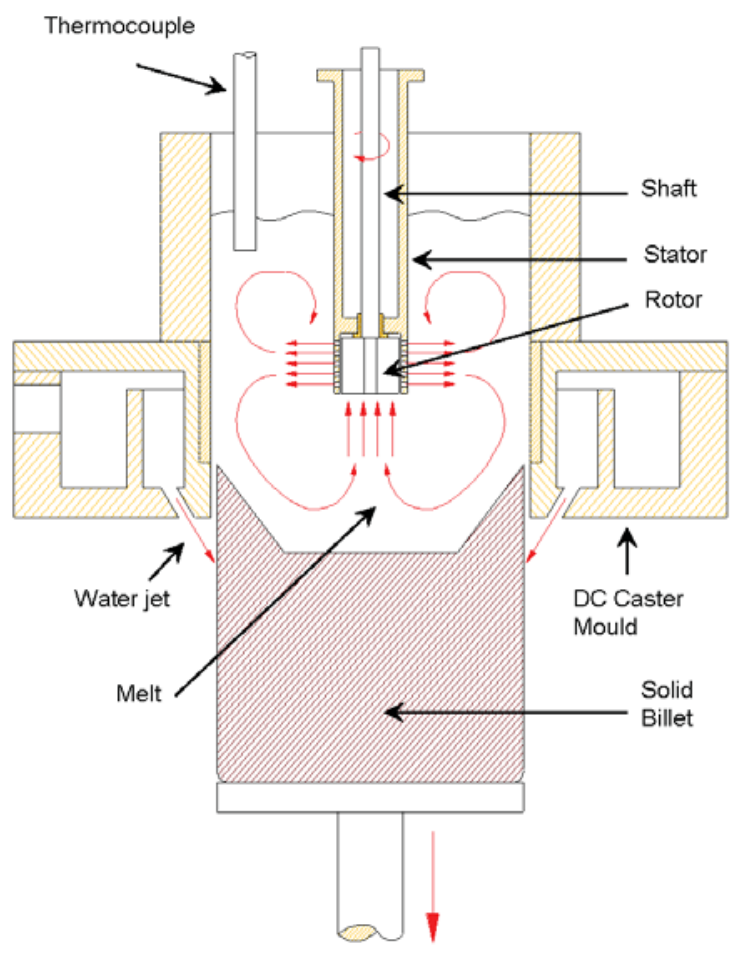

Fig. 1. Schematic illustration of the melt conditioned direct chill casting (MC-DC) process, with the high shear device submerged in the sump of a conventional DC casting mould, also showing the macroscopic melt flow pattern generated by intensive shearing.

\section{Results}

In order to demonstrate the MC-DC process on an industrial scale, castings of A6063 Al-alloy were performed on a conventional DC caster with a tilting furnace and launder/hot-top configuration, feeding a casting table fitted with a mould of $206 \mathrm{~mm}$ diameter. The DC caster was initially started at a speed of $70 \mathrm{~mm} / \mathrm{min}$ followed by $110 \mathrm{~mm} / \mathrm{min}$ with a constant water flow rate of 
120litres/min, to cast billets of $\sim 2$ metre lengths. The high shear device was mounted on top of the hot-top and preheated to $\sim 700^{\circ} \mathrm{C}$, fitted onto an electrical motor. The position of the high shear device was electrically controlled by lowering into the hot-top to be submerged in the melt and to be taken out of the melt towards the end the casting. The high shear device was lowered into the melt immediately after achieving steady state casting at the speed of $110 \mathrm{~mm} / \mathrm{min}$, and switched on to run at two different speeds, a low speed of $\sim 4500 \mathrm{rpm}$ and a high speed of $\sim 6500 \mathrm{rpm}$ for experimentation. The position or depth of the high shear device was optimised by experimenting at three variable positions. If the high shear device is placed too low, there is a risk of having it solidify with the billet or become difficult to rotate at high speed because of the increased viscosity of the solidifying melt. The position of the high shear device was experimented at different positions; P1 shearing in the hot-top, P2 just above the graphite ring and P3 within the graphite ring. The optimum shearing position was found to be P2, where it was safe to run the high shear until the end of the casting. In order to preserve the sump profile of the billet during casting and to understand the mechanism of grain structure formation under the influence of intensive shearing, a small amount of pure Zinc was poured in the hot-top which sinks immediately to the solidification front. The preserved sump profiles of the billets cast at different shearing position with variable shearing speed are shown in Fig.2.

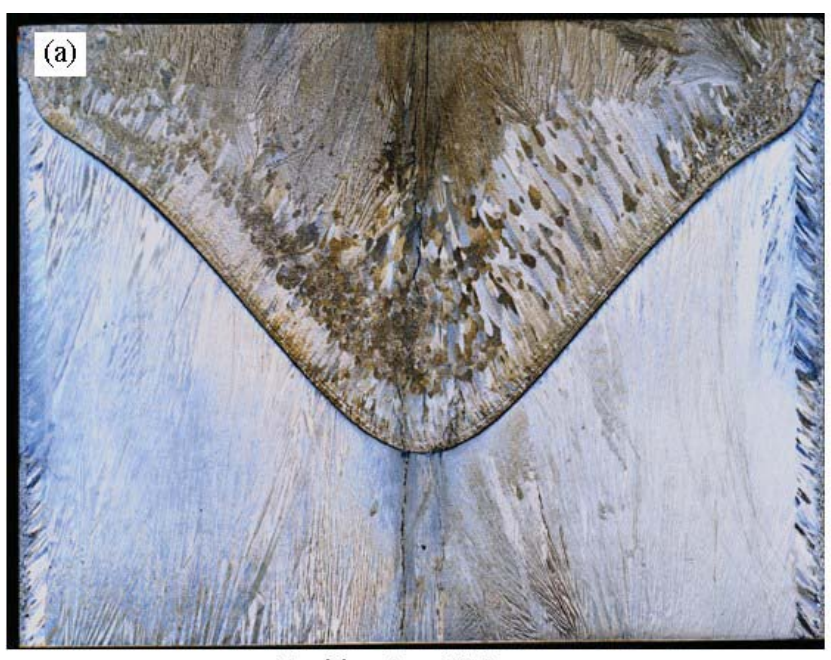

Position $1, \sim 4500 \mathrm{rpm}$

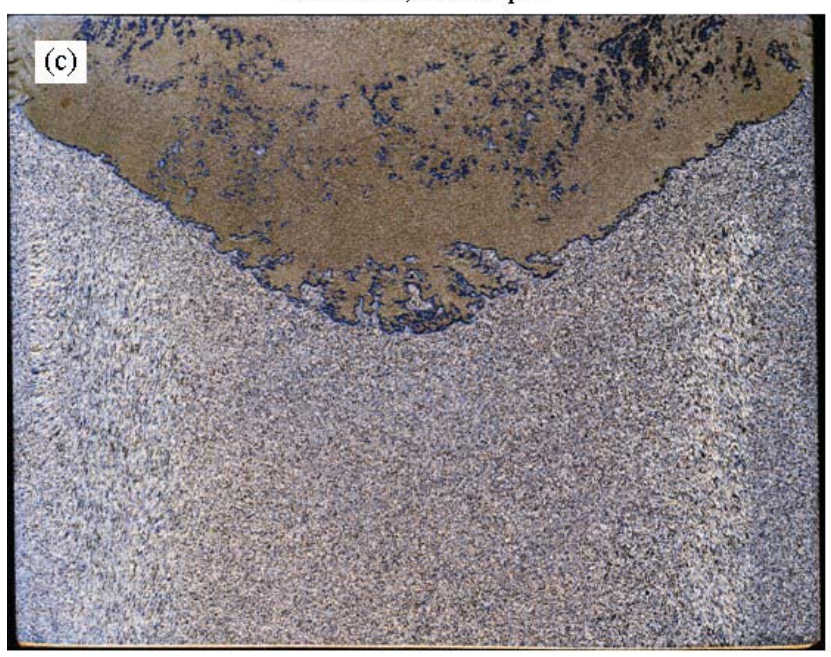

Position 2, 6500rpm

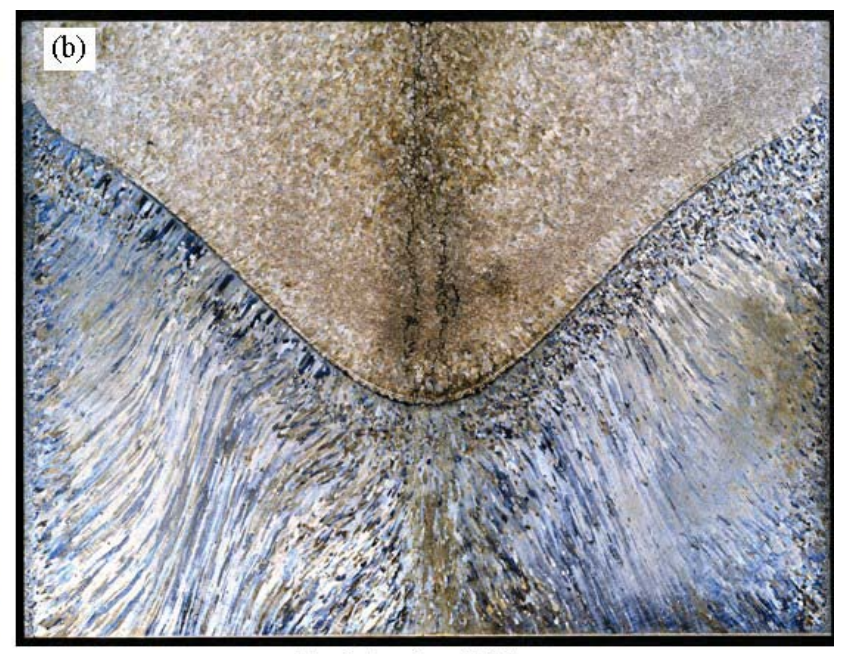

Position 2, 4500rpm

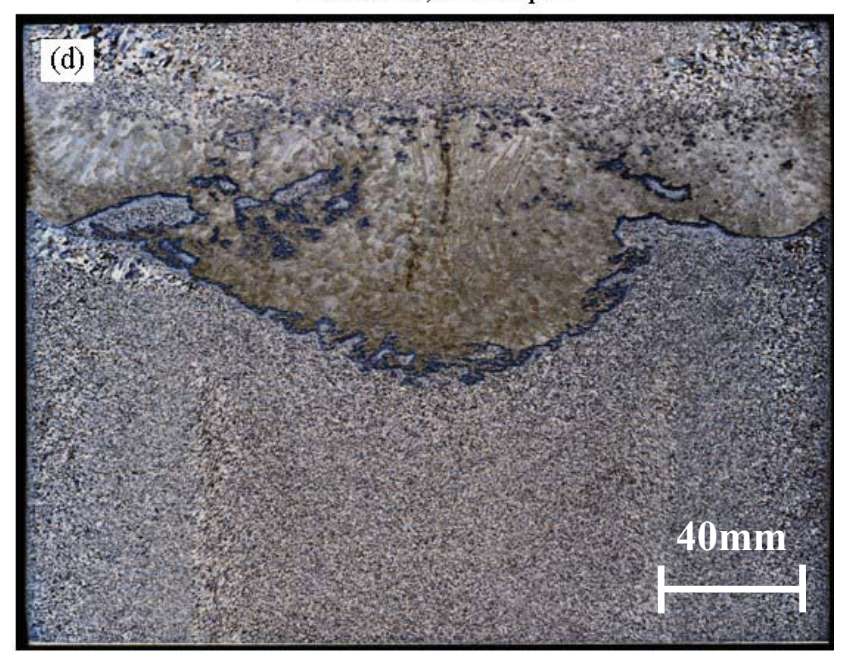

Position $3, \sim 6500 \mathrm{rpm}$

Fig. 2. Macrographs of sump profiles obtained by pouring Zn during casting MC-DC billets of A6063 alloy at different shearing positions within the DC casting mould and at low and high shearing speeds. Position 1: in the hot top, Position 2: just above the graphite ring, and Position 3: within the graphite ring. Billet diameter is $206 \mathrm{~mm}$.

It is clear from the resulting sump profiles that shearing at P1 at low speed of 4500rpm (Fig.2a), has no effect on the solidification front, where as shearing at P2 at low speed of 4500rpm (Fig.2b), 
shows that shearing starts to influence the solidification front by first reducing its depth and then having an influence on the grain structure formation. Increasing the shearing speed to 6500rpm at P2 (Fig.2c), results in further reduction of the sump depth and significant refinement of the grain structure through out the diameter of the billet. Shearing at P3 at the high speed of 6500rpm (Fig.2d), has no further influence on the sump profile and similar refinement of the grain structure is observed. Hence, the most effective and safest position for applying intensive shearing for the purpose of grain refinement of the billet is to shear just above the graphite ring in the mould. Based on the optimisation of the shearing position and shearing speed, a full billet of 2 metre length was produced by shearing at P2 at the high speed of 6500rpm. The macrograph of the cross-sectioned billet is given in Fig. 3 in comparison with a similar cross-section obtained from a billet cast without shearing, but cast with the same DC casting parameters.

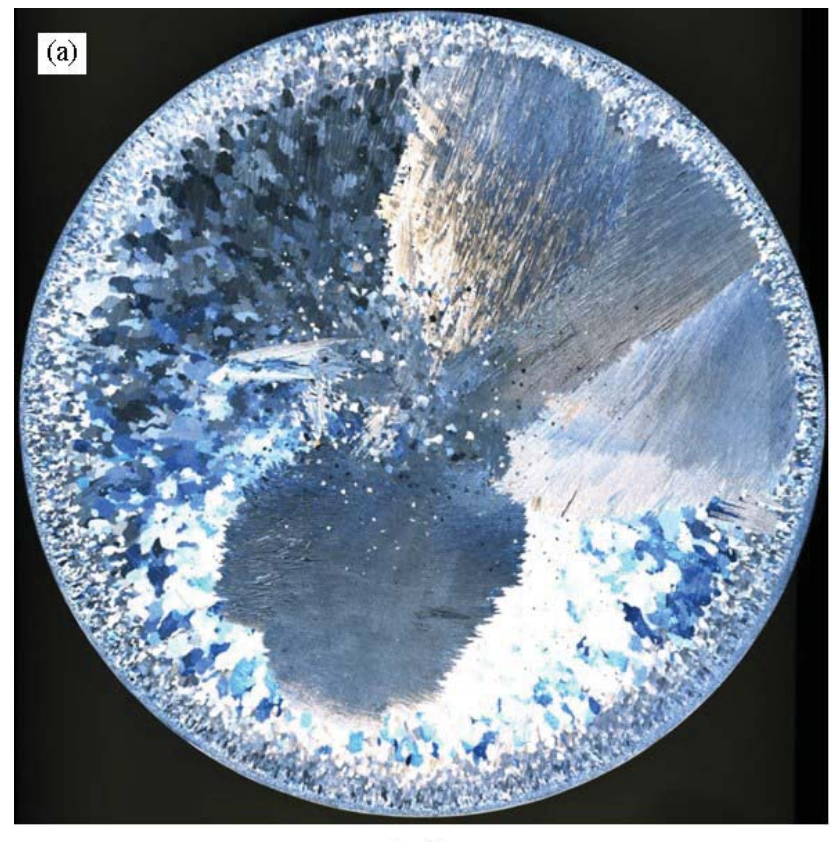

$\mathrm{DC}$

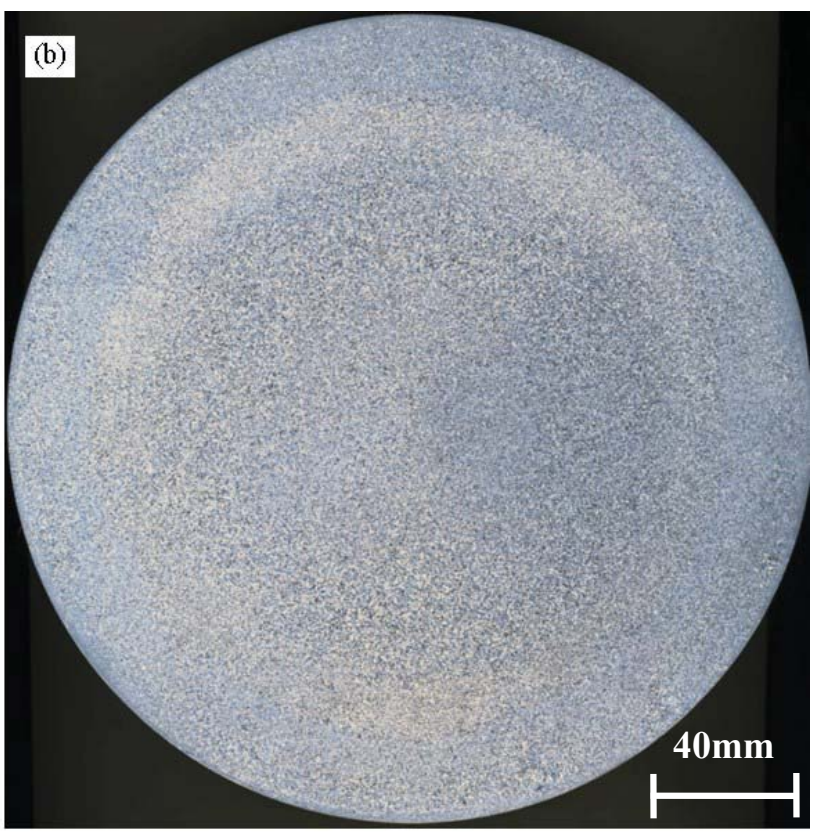

MC-DC

Fig. 3. Macro-etched cross-sections from the A6063 billets: (a) DC cast at $110 \mathrm{~mm} / \mathrm{min}$, and (b) MC-DC cast at $110 \mathrm{~mm} / \mathrm{min}$, with the same water flow rate of 120 litres/min. (Etchant: Tucker's reagent)

As compared to conventional DC casting, significant grain refinement is achieved by applying intensive shearing just above the graphite ring in the DC casting mould. In both cases, during DC or MC-DC casting no Al-Ti-B based grain refiner was added to the melt. The grain refinement achieved by intense shearing is purely a physical effect rather than a chemical effect as in the case of Ti and B inoculation. In order to further understand the mechanisms of solidification responsible for the significant grain refinement achieved upon application of intensive melt shearing during DC casting, temperature measurements were carried out during casting. Thermocouples were fixed at 3 different positions/depth inside the DC casting mould and hot-top; T1 at just above the graphite ring, followed by T2 slightly higher, and T3 in the hot-top. The temperatures were logged during DC casting and MC-DC casting in which case the shearing position was constantly maintained at P2 but the shearing speed was first set to run at 4500rpm followed by 6500rpm for the rest of the billet. The resulting temperature curves are given in Fig.4 together with a schematic of the DC casting mould showing the thermocouple positions. In the case of DC casting without shearing, the temperature variation within the mould and hot top was observed to be about $15-20^{\circ} \mathrm{C}\left(690-670^{\circ} \mathrm{C}\right)$, and this variation was maintained through out the casting. In the case of MC-DC casting, it was observed that, soon after shearing was started even at a low speed of $4500 \mathrm{rpm}$, the temperature very quickly dropped to below $660^{\circ} \mathrm{C}$ with the melt in the hot-top being at a slightly higher temperature. As the shearing speed was increased to a high speed of $6500 \mathrm{rpm}$, all temperatures were observed to be almost equal and maintained below $660^{\circ} \mathrm{C}$ (close to the liquidus) through out the rest of the 
casting. This means that at high shear speed, the melt temperature in the mould and hot-top is uniform and maintained through out the casting. The billet cast during this experiment was sectioned carefully at the correct length, in order to observe the transition zone where the shearing commenced at the low speed of 4500rpm. The macro-etched longitudinal section is given in Fig.5 together with their corresponding cross-sections.

(a) DC Casting

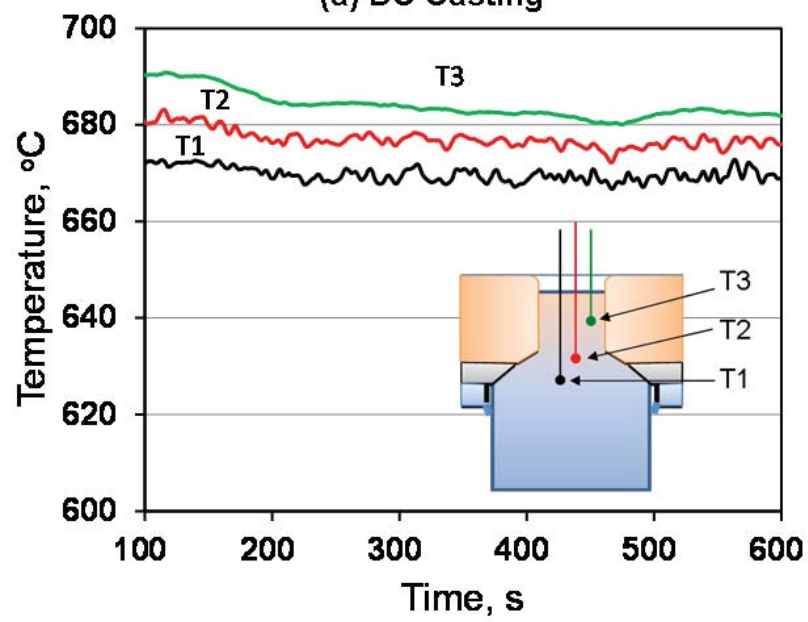

(b) MC-DC Casting

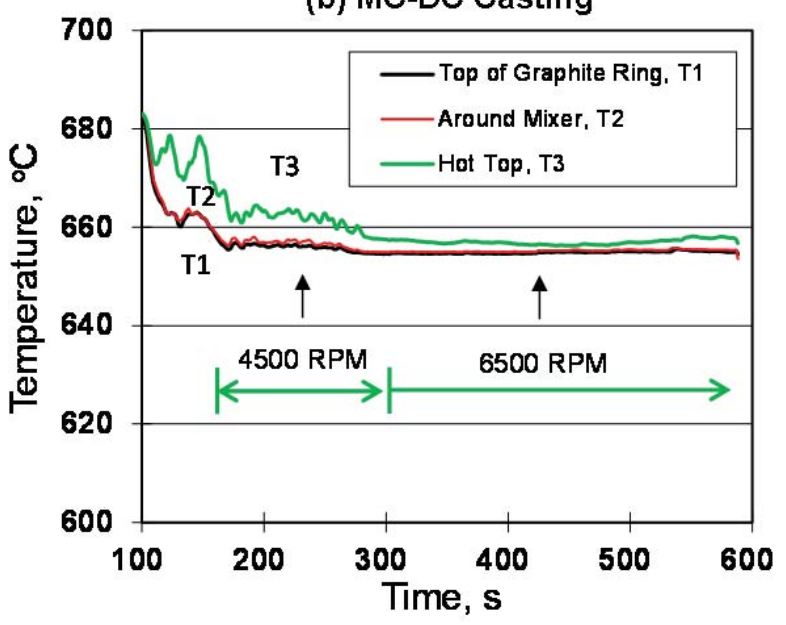

Fig. 4. Temperature data obtained from thermocouples located at different positions within the DC casting mould during casting of A6063 alloy; (a) DC casting, and (b) MC-DC casting with low and high shearing speed.
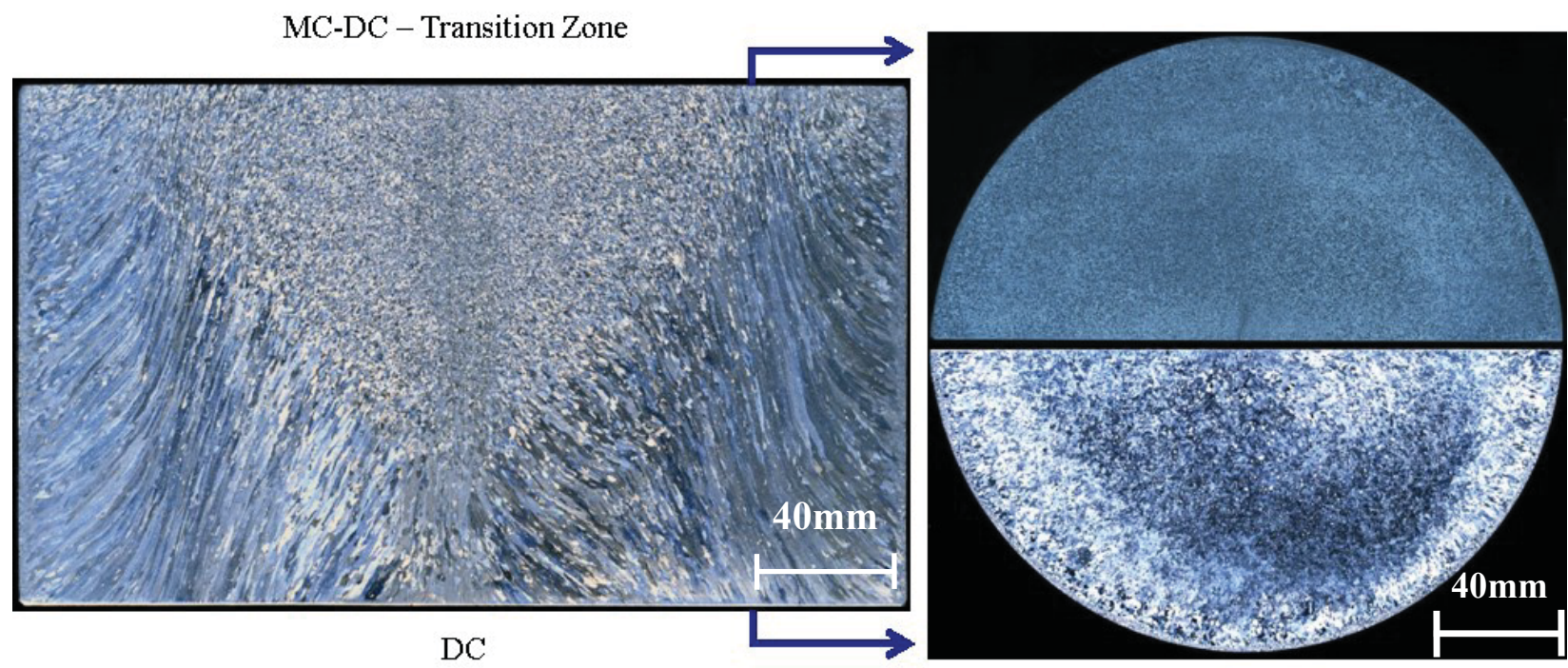

Fig. 5. Macro-etched longitudinal section of the A6063 alloy billet showing the transition zone upon application of intensive shearing at 4500rpm, together with the corresponding cross-sections from the sheared and non-sheared ends of the billet.

\section{Discussion}

Based on the grain structure formation observed from the preserved sump profiles, solidification during conventional DC casting proceeds by an advancing columnar dendritic front originating from the mushy zone. Since the sump has a positive temperature gradient from the bottom to the top, nucleation and dendritic growth both originate from the mushy zone within the sump. During MC-DC casting, the intensive shearing in the sump leads to a uniform temperature and chemical composition due to the forced convection and enhanced mass transport due to the macroscopic flow of the melt. The temperature measurement during MC-DC casting has confirmed that the melt temperature in the sump is maintained very close to or even below the alloy liquidus, which is thought to provide a unique condition for growth with a low temperature gradient and high growth velocity, which is well known to favour equiaxed grain growth according to Hunt's theory of columnar to equiaxed transition [7]. Solidification in the MC-DC casting process is believed to 
proceed by sedimentation of rosettes which initially nucleate and predominately grow within the sump with an almost isothermal temperature under severe convective melt flow. One can also imagine that these floating rosettes could have originated by dendrite arm fragmentation caused by the high shear and severe convective melt flow. It should be pointed out that, under intensive shearing conditions, no dendritic growth occurs since any grains wanting to grow are forced to maintain rosette morphology. Therefore, there are no dendrites available in the first place for any fragmentation to take place. Intensive shearing provides conditions which suppress dendritic growth and promote rosette growth and it is well known that rosettes are almost impossible to fragment [8]. In addition, the significant grain refinement can also be attributed to the enhanced heterogeneous nucleation by intensive melt shearing, which disperses oxide films uniformly through out the melt within the sump. The dispersed oxide particles can act as heterogeneous nucleation sites and therefore help in achieving grain refinement [9]. The benefits of the equiaxed solidification in the MC-DC process mainly caused by sedimentation of rosettes and enhanced heterogeneous nucleation are that it provides, grain refinement without the addition of chemical grain refiners, eliminates or reduces macro-segregation, mitigates the harmful effects of oxides and inclusions, reduces the tendency of hot tearing and formation of gas porosity, and more importantly, it provides an opportunity for DC casting alloys which are usually difficult or even impossible to cast into high quality billets.

\section{Summary}

A new MC-DC process has been developed to benefit conventional DC casting, for the production of high quality billets. The MC-DC process has for the first time, shown capability of producing industrial size billets with refined microstructure, uniform chemical composition and fewer cast defects, which is attributed to the enhanced equiaxed solidification and heterogeneous nucleation mechanisms. More importantly, the MC-DC technology is easily adaptable to an existing DC casting facility and creates a new opportunity to produce billets of alloys that are usually deemed to be not suitable for DC casting in general.

\section{References}

[1] J. Polmear; Light Alloys, Metallurgy of the light metals, $3^{\text {rd }}$ edition, 1995.

[2] D .G. Eskin; Physical metallurgy of direct casting of Aluminium alloys, CRC press, Taylor and Francis Group, 2008, p.9.

[3] D. G. McCartney; Grain refining of Aluminium and its alloys using inoculants, Inter. Mater. Rev. 34, 1989, p.247.

[4] Z. Fan, M. Xia, H. Zhang, G. Liu, J.B. Patel, Z. Bian, I. Bayandorian, Y. Wang, H.T. Li and G.M. Scamans; Melt conditioning by advanced shear technology (MCAST) for refining solidification microstructures, Int. J. Cast Met. Res., 22, 2009, p.103-107.

[5] Z. Fan, Y.B. Zuo, and B. Jiang; A new technology for treating liquid melts with intensive melt shearing, Mater. Sci. Forum, 690, 2011, p.141.

[6] Z. Fan, Y.B. Zuo and B. Jiang; Apparatus and method for liquid metals treatment, Application No.1015498.7, 2010, UK Patent.

[7] J.D. Hunt; Steady state columnar and equiaxed growth of dendrites and eutectic, Mat. Sci. Eng. A, Vol.65, 1984, p.75.

[8] A. Das, S. Ji and Z. Fan; Morphology development of solidification structures under forced fluid flow: a Monte-Carlo simulation, Acta Mater., 50, 2002, p.4571-4585.

[9] H.T. Li, Y. Wang and Z. Fan; Mechanisms of enhanced heterogeneous nucleation during solidification in binary Al-Mg alloys, Acta Mater., 60, 2012, p.1528-1537. 